Historic, Archive Document

Do not assume content reflects current scientific knowledge, policies, or practices. 



\section{Farmers Wholesale Price List}

C. S. Food Administration License No. G. $403 S 3$ SEED CORN-FARM SEEDS

ADDRES̃ AIL ORDERS

JEFEERSOY SEED CO., JEFEERSOX, IOWA.

Te make you the following prices in order to secure your early order; however, we are golns to sell our entire supply of seed at these prices, aud the prices will hold good as long as wo have a seed of our present supply on hand. After that, we will be conivelled to buy more, at bigher prices, which will naturally cost rou more than at present.

Now is the time for you to order. Take advantage of the preseut low prices, also get jonr pick and choice of all our best seed by ordering early.

ALL BAGS AND PACKING ARE FREE. This is no small item. mud should bo conshered when comparing prices. We put no dating on this price list, but reserve the right to withelvaw prices when stock on hand is sold. Send your order right in while these prices are gout.

TESTED SELD CORN-Shelled and Grarled, Guaranteed to test 91 per eent or loetler. your test.

Iowa No. 302 Prizewinner........ $\$ 3.50 \$ 2.00 \$ 1.25$

Pride of Nishna lellow Dent $3.50 \quad 2.00 \quad 1.25$

Mammoth Iowa Fellow Dent... 3.50 2.50 1.25

Iowa Gold Mine Yellow............ 3.00 1.7. 1.00

Reid's Fellow Dent …................... $3.00 \quad 1.75 \quad 1.00$

Gold Standard Ycliow

Improved Leaming Yellow ........ 3.00 1.7. 1.00

Pride of North Fellow ............ $3.50 \quad 2.00 \quad 1.25$

Queen of Nishna Yellow ....... 3.50 2.00 1.25

Legal Tender Yellow …............. $3.00 \quad \overline{1.75} \quad 1.00$

Calico or Strawberry $\ldots \ldots \ldots . . . \ldots . . .4 .400 \quad 2.50 \quad 1.50$

Giant Yellow Fodder Corn...... $3.00 \quad \mathbf{1 . 7 5} 1.00$

Giant White Fodder Corn ........ 3.00 1.75 1.00

Southern Red Cob Fodder Corn $3.50 \quad 2.00 \quad 1.25$

Eureka Ensilage Fodder Corn $3.50 \quad 2.00 \quad 1.25$

than one bushel of a variety in ear corn.

\section{CLOVER AND GRASS SEEDS}

We handle only one grade-THE BEST. Cheap seed is expensive at any price. Al prices are for JEFFERSON BRAND seed, and guaranteed to be of high grade and quality.

Bu. $1 / 2 \mathrm{Bu}$. Pk.

Clover-Medium red $\$ 16.50 \$ 8.50 \$+.50$

Mammoth Red $17.00 \quad 8.75 \quad+.7 .1$

Alsike $\begin{array}{lll}17.00 & 8.10 & 4.7 . \\ 18.00 & 9.50 & 5.00\end{array}$

Alfalfa, Nebraska, Kansas or

$\begin{array}{llll}\text { Dakota } & 16.50 & 8.50 & 4.50\end{array}$ White Blossom Sweet Clorer 12.00 $6.50 \quad 3.50$

Crimson Clover .......................... 9.00 $4.75 \quad 2.50$

White or Dutch Clorer ......... 39.00 1S:0 9.75

TIMOTHIY …............................ $4.50 \quad 2.50 \quad 1.35$

Timothy and Alsike Mixture... $7.00 \quad 3.7 .52 .00$

MILLET, Golden, N. or S..... $2.50 \quad 1.35 \quad .75$

Siberian Millet …....................... $3.00 \quad 1.75 \quad 90$

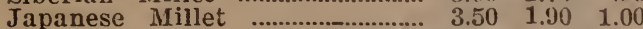

Per 100 lbs. 50 lbs.

BILIION DOLLAR GRASS $\$ 10.00$ \$6.00

SUDAN GRASS ....................... $12.00 \quad 6.50$

\section{LIGHT GRASSES}

Kentucky Blue Grass Meadow Fescue or Eng. Bluegrass $30.00 \quad 4.50$ Rye Grasses

Tall Meadow Oat Grass..................... $85.00 \quad 500$

Red Top Grass ..................................... 15.00 2.60

Johnson Grass

Bermuda Grass

Bromus Inermis

Lawn Grass

$3000 \quad 4.50$

60.00 8.50

$27.00 \quad 3.90$

$4000 \quad 6.00$

Pasture Mixtures
SEED YOTATOES, (See page 38 ) per bu., $\$ 3.50$

SEED GRAIY

10 bu. 5 bu. 1 bu.

Bu. Bu. Bu.

OATS-New Alberta $\$ 1.00$ \$1.05 $\$ 1.10$

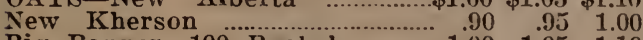

Big Banner $\rightarrow 100$ Bushel ............. $1.00 \quad 1.05 \quad 1.10$
1311. 1/213u. 1'k

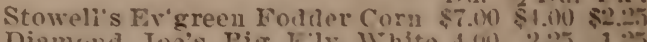
Diamund Joe's Big Fily White 4.01 2.25 $1: 25$ Iowa silver vine White ........ 3.50) 2.(h) 1.20 Johusun Connty White ............. 3.10 1.75 $1 . n 0$ Wallace's Favorite White ........30 300 St. Charles White ............. 3.50 2.(4) 1.27 Boone county White ............... $3 .(\mathrm{k}) \mathrm{1.75} 1.010$ Imperial Red Cob White ........ 3.51 ith 1.00 Hickory King White ................ 400 (2.25 1.25 Blondy Butcher ........................... 4.00 2.25 1.25 I.ongfollow Iellow Flint ........ 4.00 2.25 1.25 Iibble's Fellow Flint ...............4.4.00 2.2. 1.25 Farly Canadian Flint ................ 4.010 2.251 .25 5-bushel lots or more, aic per bushel less. el to abore prices. No orders filled for less Inwa No. 103

10 เи. 5 ดแ. 1 นแ. Bu. Bu. Bu.

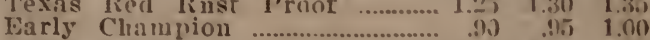
Silver Mino

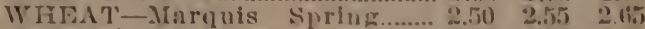
Macaroni or Duram spring.... $\begin{array}{ccc}2.50) & 2.55 \\ 2.51 & (1.5)\end{array}$ Malakoff Fall Wheat Turkey Rrd Fall Whest....... 250 2.550 SIPLTZ OR FMIIER

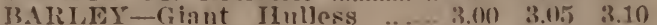
Success Bearilless .................... 310 3.05 3.10

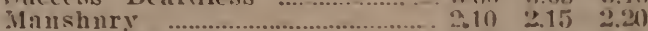

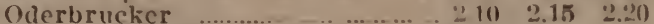
RYE-Mammoth Siring -... - 2. White Wonder Whuter .............. 2.5) 2.5.5 2.(t)

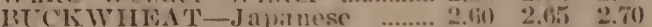
Silver IInll ....................................(1) 2.05 2.70

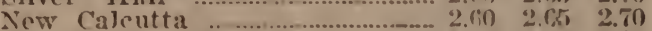
WIAX-New Russtan ................ (\$.00 6.05 (5.10 Minmesota or Primroso ........... (i.00 8.0. 8.10 COTTON SEED-13rown's No. 1 3.00 3.10 3.15 Jong Staple …................ $3.10 \quad 3.15$ Texas Triumnh ……................. RUSSIAN SUNFLOWIR SIII), \$1" per Civt.

\section{FORIGE ANO TODDTIR CIOPS}

('wt. Bu.

Jerusalem Corn ................................... \$11). (n) $\$ 900$

MIilo Maize ............................................. $3.50 \quad 2.10$

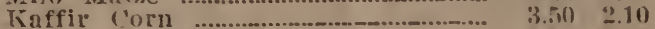

CANE-Orange or Amber ................ 4.00

Snrghum, Orange …......................... $10.00 \quad 5 .(0)$

Dwarf Essex Rape ....... 12.00 (i.10)

Broom Corn-Standard Evergreen 4.0? 2.11) Feterita …..................................... 1.(u) Pencila ria Sand Vetch ......................................... 20.(1) 10.50 Spring Veteh ................................. 10.00 5. Soy Peans ........................................ 7.00 4.00

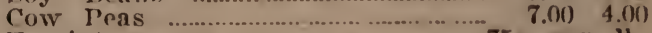
Teosinte 75e per ib. 


\section{Wholesale Garden Seed Prices}

\section{[Subject to stock unsold]}

In quotins the following wholesale prices on Garden Seeds, we offer you the best of fresh, new crop Garden Seed, at prices much lower than you ean secure them at home.

In order to take advantage of these prices, your total order must amovit to 5 pounds or more, of the different seeds. Not 5 pounds of any one variety but a total order of 5 pounds or more. Garden Seed prices in our catalog are quoted lost-paid. rhese are not. If you wish seeds sent by parcel post, add postage at the rate of $5 \mathrm{c}$ per pound, or see parcel post rates oil page 3 of our Catalog, otherwise seeds will be sent by express or freight at your expense.

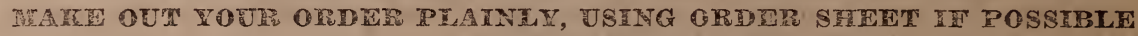

This will sare trouble and delay. ALT SEED GUARANTEED TO GROW OR REPLACED FRED OF CHARGE. ALI BAGS AND PACKING FREE.

Variety

ASPARAGUS

Columbian Barr's Nammoth..... Paimetto

Corover"s Coloseal

\section{BEANS}

\section{Bountiful}

Stringless G'n Pod Refugee

Loilgtellow

Longr Iel. 6 Wks

Imp. Red Valentine

Wardwell's $K$. W. Dwart Black W. German Black W. Davis White Wax Golden Wax

New Kidney Wax Burpee's B. Lima Henderson's Bush Lima

Fordhoois B. rima Large White Lima Dreers' B. Lima... Kentucky Wonder oid Homestead... Lazy IVife

Dritch Case Knire Cut Short ox Cornfield

Hoticultural

Giaut Podded I

King of G'd'n Iima

White Tapary

Choice Navy

Burlingame Field..

\section{BEETS}

\section{Tb. $1 / 2$}

Crosby's Hgyptian

Dark Red Egypt.

Early Eclipse.......

Eariy Dark Beauty

Detroit Dark Red

Early Blood Tnp.

Market Gardenex..

Crimson Globe.....

Swiss Chard ........ 1.15

Mammoth Long Red Mangel

Golden Tankard Mangel

Giant Half Sugar

Half Sugar Rose..

Klein Wanzelben

Sugar ….........

Vilmorin Sugar....
White French Sug.

\section{Price \\ Furiety}

BRUSGELS

$\mathrm{Lb} 1 / 21 \mathrm{~b} 1 / 4,1 \mathrm{~b}$

SPROUTS

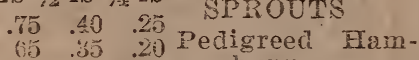

$\begin{array}{llll}65 & .35 & .20 & \text { Pedigmed } \\ .65 & .35 & .20 \text { burg }\end{array}$

20 burg …........... 2.501 .50 Dalkeith …........... 2.501 .50 CABBAGD

Lb. $1 / 210.1 / 413$

Price

Variety

Price

Peer 0' Day Mammoth White... $12 \quad .12$.1: 11 $\begin{array}{llll}\text { C'ntry Gentleman } & .10 & .11 & .11 \\ & & .11 & .12\end{array}$

.80 Stowell's Evergr'n $\quad .10 \quad .11 \quad .12$ $\begin{array}{lllll}.80 & \text { Black Mexican..... } & .10 & .11 & .12\end{array}$ .80 Early J'sy W'field 2.50 1.35

1b. 1b. 1h. Wash'ton Wakefl'd 2.501 .35

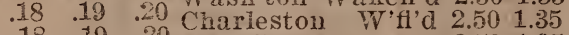

$\begin{array}{lll}.18 & .19 & 20 \\ 18 & \text { All Seasons ....... } 2.50 & 1.35\end{array}$

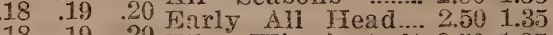

$\begin{array}{llll}18 & .19 & 20 \\ 18 & 19 & \text { Winnigstadt } 2.50 & 1.35\end{array}$

.18 .19 .20 Sopenbaren Mkt. 3.001 .60

18 ig .20 lceal Iute .......... 2501.35

20 Large Dirum H'd 2.501 .35

.20 Autimn King … 2501.35

.20 Farly Flat Dutch 2.501 .35

.20 Holland Cabbage.. 3.00 1.60

20 Fate Wnt Dutch 2.501 .35

20 Sure pead …......- 2501.35

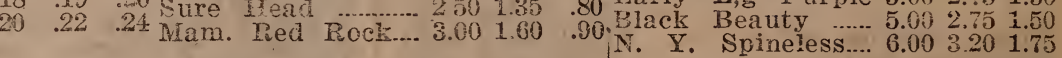

4 POP CORN Ib. 1b. 1b.

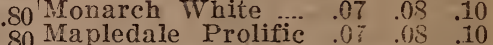

.80 Hulless

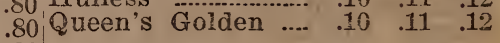

.80 CELERY Lb $1 / 2151 / 410$ .90 Golden Blanching $3.06,1.851 .00$ .80 White Plume.......... 2.50 1.3.5 .50 .80 Giant Pascal ........ 2.001 .25 .8v .80 Celeriac …................ $3.50 \quad 2.00 \quad 1: 10$ .80

.20 EGG PLANT Lb $1 / 2$ ib $1 / 4$ Ib .80 Narly L,g Purple 5.002 .751 .56 $\begin{array}{lllll}.80 & \text { Narly L,g Purple } 5.00 & 2.75 & 1.56 \\ \text { Black Beauty ….. } & 5.00 & \mathbf{2} .75 & 1.50\end{array}$ $.20 \quad .22 \quad .24$

$.20 \quad .22$

$.20 \quad .22$

$.20 \quad .22$

$.20 \quad .22$

$.20 \quad .22$

$.20 \quad .22$

$.20 \quad .22$

$.20 \quad .22$

$.20 \quad .22$

$.20 \quad .22$

$.16 \quad .17$

$.16 \quad .17$

.24 CARROMS

Ib. $1 / 2161 / 4$ Ib

KOHL RABI Lb $1 / 21 \mathrm{~b} 1 / 4 \mathrm{lb}$

.24 Golden Beanty - $\quad .80 \quad .45 \quad .25$ Early Pur. Vienna $2.50 \quad 1.35 \quad .70$

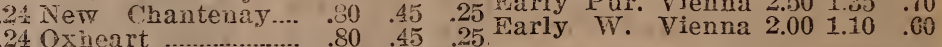

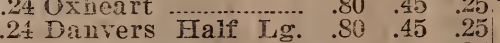

24 Trp Orange

.24 E'iy Scarlet Horn Mastadon

ENDIVN

$\mathrm{Lb} 1 / 2$ ib $1 / 4$ ib

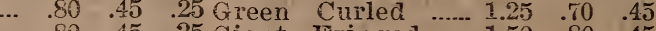
.24 Long Lemon

.24

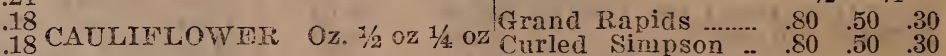

LETTUCP

Ib $1 / 2$ ib $1 / 4$ lb

$\begin{array}{llllllll}.18 \text { Dan. Dry Weather } 2.00 & 1.10 & .60 & \text { Black Simpson } & .80 & .50 & .30\end{array}$ $\begin{array}{lllllllll}\text { Early Erfurt ….... } 2.00 & 1.10 & .60 \text { Cal. Cream Butter } & .80 & .50 & .30\end{array}$

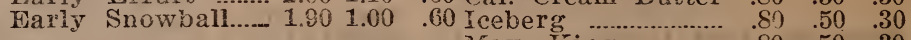

$\begin{array}{lrlllll}1 / 210.1 / 4 & 10 . & .50 & .50 & .30\end{array}$

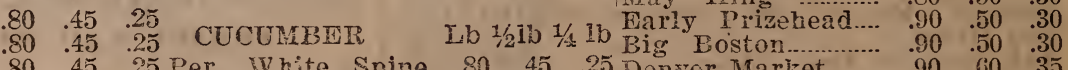

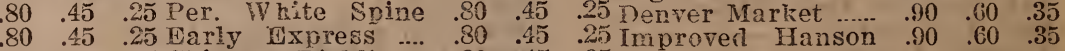

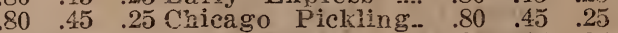

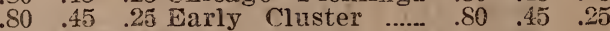

.45 .25 Davis Perfect

$25 \mathrm{Imp}$. Long Green

$.35 \mathrm{Cool}$ and Crisp...

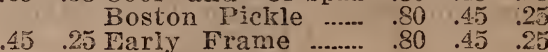

$.80 \quad .45$

$.80 \quad .45$

$\begin{array}{lll}.80 & .45 & .25 \\ .80 & .45 & .25\end{array}$

$\begin{array}{lll}.75 & .45 & .25\end{array}$

$.80 \quad .45$

.80 .45

$80 \quad 45$

25 SWEET CORN

Cwt 50 lbs 10 lbs Birrell Gein

$\mathrm{Lb} 1 / 2 \mathrm{lb} 1 / 4 \mathrm{lb}$

25 MUSTARD

$.90 \quad .50 \quad .30$

$\begin{array}{lllll} & \text { Southern Giant...... } & .90 & .50 & .50\end{array}$

\section{MUSTMELON}

Lb $1 / 2$ lb $1 / 4$ lb

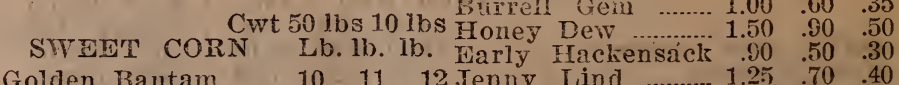
$\begin{array}{lllllllllll}5 & 25 & \text { Early White Cory } & .12 & .13 & .14 \mathrm{Gem} \text { of } & .12 \mathrm{Gen} & . . . & 1.35 & .70 & .40\end{array}$ $\begin{array}{llllllllll}45 & 25 \\ \text { Early Minnesota.... } & .12 & .13 & .14 \text { Emerald Gem } & . . . . . . & .90 & .50 & .50\end{array}$

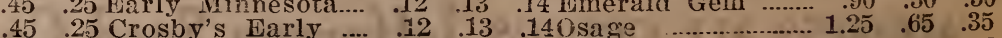


You don't have to use this order sheet. You can order any way you wish, but using this will save us both some bother and lessen mistakes. Better keep an exact copy for future reference. Be sure to give your NAME and ADDRESS.

\section{ORDER SHEET FOR SEEDS}

Every order, large or small, will receive prompt and careful attention.

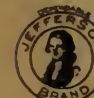

All members of one family should order under one name.

Name

Post Office

Shipping Point

State County

R. F. D. No.

P. O. Box No.

Street No.

Date of Order

Amount enclosed

\section{HOW SHALL WE SHIP}

Parcel Post

(Postage Enclosed)

Name of Express Co.

If there is no freight agent at your shipping point, it will be necessary for you to send money to prepay freight charges. If there is an agent, you can pay freight when the shipment reaches you. It is only necessary to prepay charges where there is no agent at your station. The charges are the same.

In case there has been either a rise or fall in the price of such seeds as are subject to market changes, we will always ship seed to the full market value of the money sent.

SATISFACTION GUARANTEED OR MONEY REFUNDED. It is distinctly understood and agreed that any seeds or other goods which are not satisfactory may be returned to us within ten days after you receive them and we will refund the money paid for same. We will not be satisfied unless you are satisfied, but we do not and cannot in any way warrant the stand nor the crop from any seed for they depend on many conditions which are beyond our control.

\begin{tabular}{|l|l|l|l|l|}
\hline Quantity & Name of Seeds and Articles Wanted & Price & Total \\
\hline & & & & \\
\hline & & & & \\
\hline & & & & \\
\hline & & & & \\
\hline
\end{tabular}




\begin{tabular}{|l|l|l|l|}
\hline & & & \\
\hline & & & \\
\hline \\
\hdashline & & & \\
\hline & & & \\
\hline \\
\hline
\end{tabular}

If you get more than one Catalog please hand it to some friend.

We shall consider it a special favor and will be pleased to include in your order a packet of our White Icicle Radishes, if you will write here the Names and Addresses of your neighbors who usually send for their seeds. Please do not write anything but Name and Address in the space below.

Name

Post Office

State R. F.D 


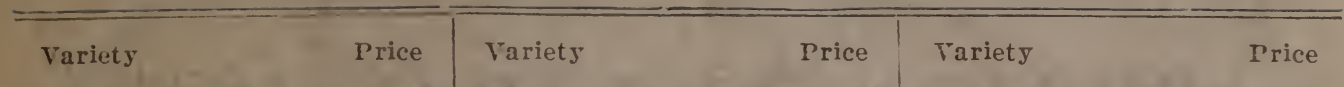

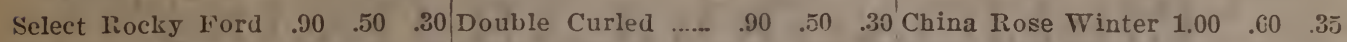

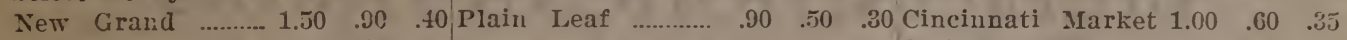
$\begin{array}{lllllllllll}\text { Green Nutmeg } & . . . & 1.00 & .60 & .35 & .35\end{array}$

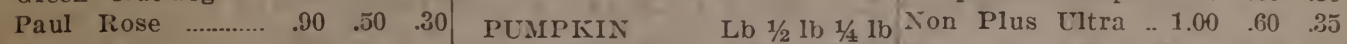
Sugar or Sweet..... .SO $.45 \quad .25$

WATERMELON $\mathrm{Lb} 1 / 2 \mathrm{lb} 1 / 4 \mathrm{lb}$ Kentucky Ficld.......

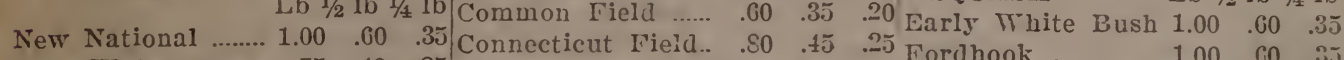

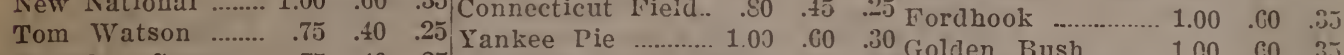

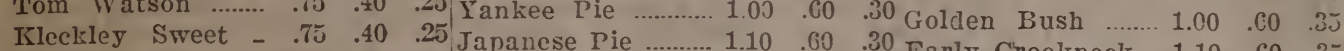

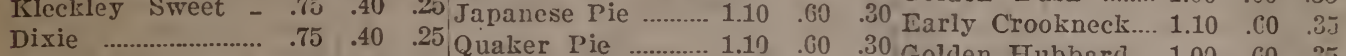

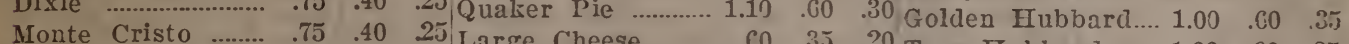

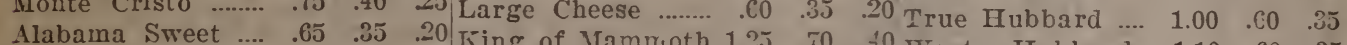

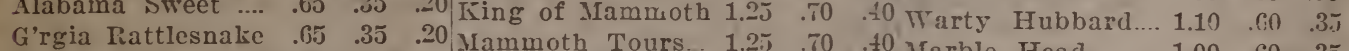

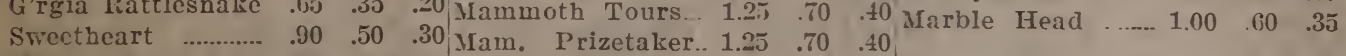
$\begin{array}{llllll}\text { Black Diamond .... } & .65 & .35 & .20 & .30 & \end{array}$

New Champion $\quad$.... $1.00 \quad .60 \quad .35$

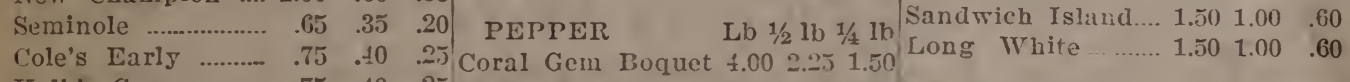

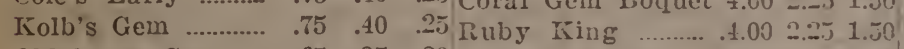

Oklahoma Gem … $.65 \quad .35 \quad .20$ Chinese Giant … $5.00 \quad 3.09200)^{\prime}$ SPINACH L L 1/2 lb $1 / 4$ lb

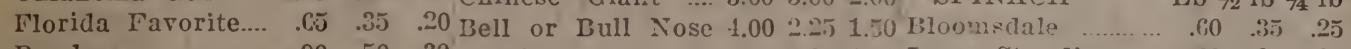

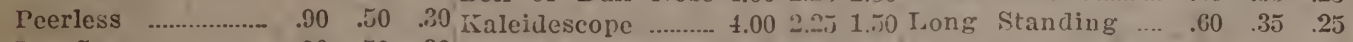
Ice Cream …........ $\quad .90 \quad .50 \quad .30$ Sweet Mountain .... $500 \quad 3.001 .60$ Light Icing …....... .75 $\quad .45 \quad .25$ Red Chili ................ $5.003 .00 \quad 1.60$

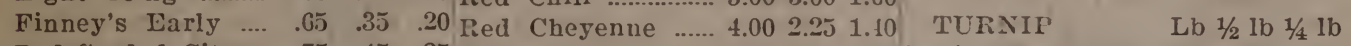

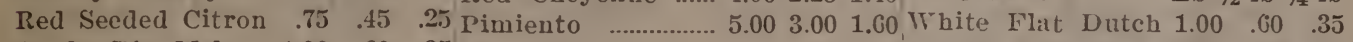
$\begin{array}{llllllllll}\text { Apple I'ic Melon.. } 1.00 & .60 & .35 & \text { Purple Strap Lenf } 1.00 & .60 & .35\end{array}$ $\begin{array}{llllll}.50 & \text { Early White Milan } & 1.50 & .50 & .50\end{array}$ OKRA Lib $1 / 2 \mathrm{lb} 1 / 4 \mathrm{lb} \quad$ Cwt. $50 \mathrm{lbs} 10 \mathrm{lbs}$ Purple Top Milan $1.50 \quad .90 \quad .50$ $\begin{array}{llllllllll}\text { White Velvet } & . . . . . . . . & .60 & .35 & .20 & \text { PEAS } & \text { Lb. 1b. 1b. Early Snomball... } 1.00 & .60 & .35\end{array}$

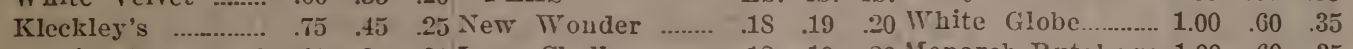
$\begin{array}{lllllllllll}\text { Perkins Long Pod } & .60 & .35 & .20 \text { Iowa Challenge } & . . . & .18 & .19 & .20 \text { Monarch Rutabaga } 1.00 & .60 & .35\end{array}$ First and best ...... . . is .19 .20 Amer. Rutabaga.... $1.00 \quad .60 \quad .35$

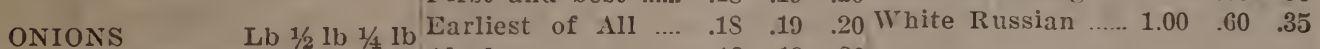

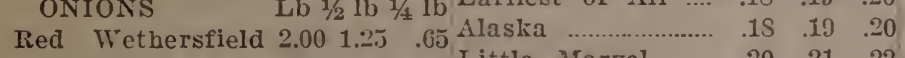

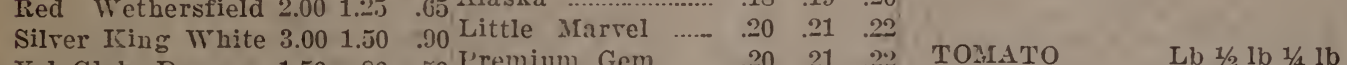

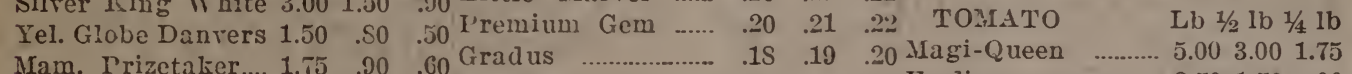

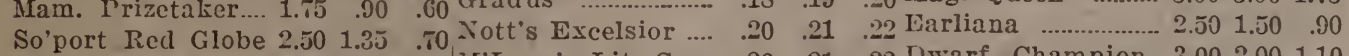

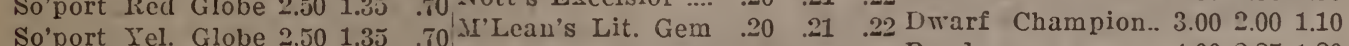

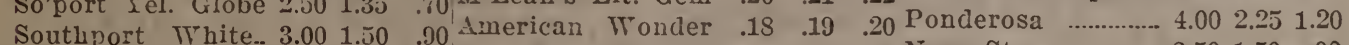

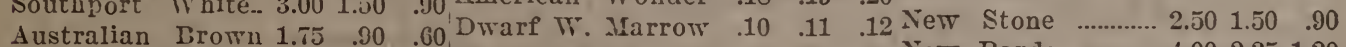

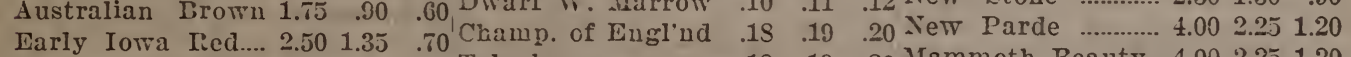

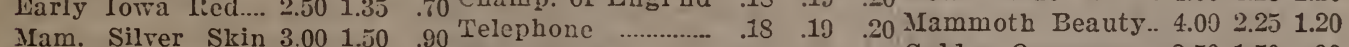

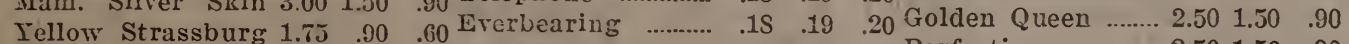

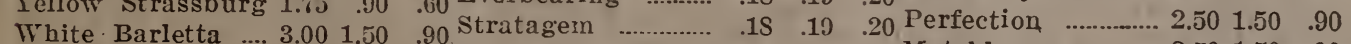

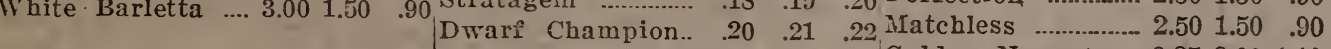
Golden Nugget .... 3.252 .001 .10 Straw'ry or Husk 3.002 .001 .10 ONION SETS Lb. lb. 1b. STEET PEAS Lb $1 / 2$ lb $1 / 4$ lb Ground Cherry …... 3.002 .001 .10

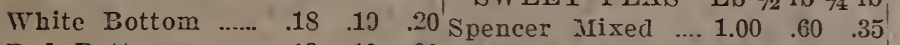
$\begin{array}{llllllll}\text { Red Bottom } & \ldots . . . \ldots . . . & .18 & .19 & .20 \text { Eckford's Mixed.... } & .75 & .40 & .25\end{array}$ $\begin{array}{lllllll}\text { Yellow Bottom } & \ldots . . & .18 & .19 & .20 & .0 & \end{array}$

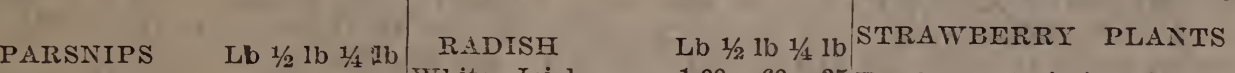
Hollow Crown $\quad . \quad .80 \quad .45 \quad .25$ White Icicle ....... 1.00 $1.60 \quad .35$ Ererbear Varieties....\$ $.50 \quad \$ 3.50$ $\begin{array}{llllllllll}\text { Guernsey } & \text {................... } & .80 & .45 & .25 & \text { Luctle Fed Wonder } 1.10 & .60 & .35 \text { Standard varieties .... } & .35 & 2.50\end{array}$ Scarlet Turnip .......1.00 $.60 \quad .35$ Rhubarb Roots .......... $1.50 \quad 5.00$ $\begin{array}{llllll}\text { Erench Brealfast.. 1.00 } & .60 & .35 \text { Asparagus Roots } & \ldots . . . . . & .60 & 3.00\end{array}$ PARSLEY Lb $1 / 2 \mathrm{lb} 1 / 4 \mathrm{lb}$ Improved Chartier 1.00 .60 . 35 Horse Radish Roots $.75 \quad 4.00$ $\begin{array}{lllllllllll}\text { Moss Curled } & . . . . . . . & .90 & .50 & .30 \text { California Winter } 1.00 & .60 & .35 \text { Everbearing Raspb's } 1.25 & \mathbf{7 . 5 0}\end{array}$ 


\section{"BILLION DOLLAR GRASS"}

The Most Valuable of All Annual Grasses Grown

WSPECIALLY VALUABLE FOR SHLO AND FOR FEXDING GREEN-BETTER THAN THE BEST CORN FODDER

From Japan, that mysterious country, which is furnishing (and has furnished in the past) many rale farm seeds, comes this remarkable grass-remarkable in a hundred different ways, for there is nothing known to man today that is more luxuriant, more prolific, more marvelously rich and vigorous in growth than Billion Dollar Grass. It will produce a hay crop in from six to ten weeks, anywhere, and if sown the first of May will be ready to cut the middle of July for hay, attaining the height (according to the richness of the soil and warmth-) irom 5 to $71 / 2$ feet. It is pre-eminently the grass to sow if you are going to be short on hay for your horses, mules, cattle and other stock.

BETTER THAN CORN FOR SILO-Cows with both Billion Dollar Grass and corn before them will take the Billion Dollar Grass first and consume it without waste; when put upon Billion Dollar Grass they increase in milk and fall off when feed is changed to corn. An ideal ensilage mixture is made of two parts Billion Dollar Grass and one part of Soja Beans, or Cow Peas, mixed when filling the silo. This mixture forms a complete balance ration for milch cows without grain, oil cake, etc.

CCLTURE-Everybody reading the above regarding this wonderful grass will wish to know its best method of cultivation. If you wish very fine leafy hay, and have strong, rich ground, sow at the rate of twenty pounds per acre, but if your soil is but moderately rich. then sow at the rate of fifteen pounds per acre. This latter is the amount we ourselves sow, and find same very satisfactory. It can be sown at different times of the year, especially if you wish same for pasturage, or if you are short of hay. It can be sown in April, in May, in June, in July, and even in August, and will return glorious, heavy crops.

FEEDING VALUE-Here is wiere Billion Dollar Grass comes into play. Prof. Williams of the United States Department of Agriculture says "Already widely grown as a hay crop, it deserves more general use for soiling, as this grass is of particular value for feeding to dairy cattle, young stock and sheep."

FOR FEEDING GPEEN-All rich, luxuriant growing grasses and forage plants must be fed in the green state moderately at first. Billion Dollar Grass is one of those rich, magnificently luxuriant growing grasses : therefore you should turn in hogs and cattle and sheep and horses at first moderately, after they have had a good feed somewhere else, and then gradually astom them to this. It is on the same plan as hungry stock turned into rich clover, they would find harm. Turn them in at first moderately, and you can soon keep them permanently.

FOR SEED-Let Billion Dollar Grass get thoroughly ripe; cut and thresh as rou would timothy. The seed is rich and nutritious. Poultry, geese, and turkeys all relish Billion Dollar Grass in the green state, and keep healthy and fresh on the seed thereof when fed during the fall and winter months.

Hogs and their families. The hogs grunt and give that contented swag of the tail when they can pasture on Billion Dollar Grass, and then if you wish to fatten them, give them rations of the seed. Remember, this grass will produce from forty to sixty bushels of seed per acre. This fed, either ground or coarse, makes excellent food, although it is inclined to be slightly laxative.

Cows, calves and other stock look with pleasure on the Billion Dollar Grass pasture and feed in contentment upon its luxuriant swath and glory in Billion Dollar Grass hay, especially if fed along with Cow Pea hay or Pea Oat hay or grain. Try it this season to make hay out of it, fill your silos therewith and watch the cattle eat it.

Horses, mules and the like jump over a four-foot fence to get at the Billion Dollar Grass, and they will fatten and keep healthy thereon. They will eat the hay as eagerly as cattle.

A REMARKABLE GROWER-It is truly the most marrelous grower, the most luxuriant stooler that we have ever seen. Imagine a field sown to Billion Dollar Grass, with every spear of it over six feet tall, yielding from 26,000 to 50,000 pounds of as rich, green fodder as the world has ever seen. Men talk about hard times, but positively hard times must vanish on every farm where Billion Dollar Grass is sown. You can get two or three rich crops from on every farm whes if you sow early you will get from 26,000 to 50,000 pounds of fodder, which you can put in your silo or cure for hay. Billion Dollar Grass will quickly spring up again and give you another yield that will astonish the country. This can again be cut for hay; then the field will give you pasturage all fall, and horses and hogs and sheep and cattle and poultry will eat it greedily.

IF YOU SOW PLENTY OF BILLTON DOLLAR GRASS YOUR WIFE CAN DRESS IN SILK AND SATIN AND YOU CAN TAKE YOUR FAMILY AND FRIENDS OUT RIDING IN AN AUTOMOBILE! YOU CAN ROLL IN WEALTH AND LUXURY. BILLION DOLLAR GRASS IS A GOLD MINE TO THOSE WHO SOW IT ABUNDANTLY.

PRICE OF BILLION DOLLAR GRASS-Postpaid: Package 10c, pound 30c, three pounds $75 \mathrm{c}$. Express or Freight, 15 pounds (enough to sow one acre) $\$ 2.50,30$ pounds $\$ 4.75,50$ pounds $\$ 6.00,100$ pounds $\$ 10.00$. Bags free.

\section{JEFFERSON SEED COMPANY}

